\title{
Implementation of the VMM ASIC in the Scalable Readout System
}

\author{
M. Lupberger ${ }^{\mathrm{a}, *}$, L. Bartels $^{\mathrm{b}, \mathrm{a}}$, F. M. Brunbauer ${ }^{\mathrm{a}, \mathrm{c}}$, M. Guth $^{\mathrm{d}, \mathrm{a}}$, S. Martoiu ${ }^{\mathrm{e}}$, \\ H. Müller ${ }^{\mathrm{a}}$, E. Oliveri ${ }^{\mathrm{a}}$, D. Pfeiffer ${ }^{\mathrm{a}, \mathrm{f}}$, L. Ropelewski ${ }^{\mathrm{a}}$, A. Rusu $^{\mathrm{e}}$, P. Thuiner ${ }^{\mathrm{a}}$ \\ ${ }^{a}$ CERN, Geneva, Switzerland \\ ${ }^{b}$ Georg-August-Universität Göttingen, II. Physikalisches Institut, Göttingen, Germany \\ ${ }^{c}$ Technische Universität Wien, Wien, Austria \\ ${ }^{d}$ Albert-Ludwigs-Universität Freiburg, Physikalisches Institut, Freiburg, Germany \\ ${ }^{e}$ Horia Hulubei National Institute of Physics and Nuclear Engineering, Magurele, \\ Bucharest, Romania \\ ${ }^{f}$ European Spallation Source ERIC, Lund, Sweden
}

\begin{abstract}
The Scalable Readout System (SRS) developed by the RD51 collaboration is a versatile and multi-purpose approach, which is used with different front-end chips to transfer data from detectors to computers. Targeting mainly micropattern gaseous detectors, the system is also applicable for silicon strip or pad detectors. The most frequently used front-end chip today is the APV25, originally developed for the CMS pixel detector. In the scope of the ATLAS New Small Wheel upgrade, a new front-end chip, the VMM, is developed, which has significantly improved specifications compared to the APV25.

We report on the implementation of the VMM in the Scalable Readout System carried out by the RD51 collaboration in the framework of a detector project related to the European Spallation Source ERIC. Due to the hierarchical design of the Scalable Readout System, only specific parts of the readout chain need to be adapted or designed, which is the carrier board for the front-end chip, an adapter card that connects to the common hardware of the system and the firmware for a field programmable gate array. In addition, we have developed dedicated software for slow control, data acquisition and online monitoring. The
\end{abstract}

\footnotetext{
${ }^{*}$ Corresponding author

Email address: michael.lupberger@cern.ch (M. Lupberger)
} 
readout system has been tested in the laboratory and in particle beams and we present results which proof the functioning of the system, even though it is still in a prototype state.

Keywords: Data acquisition, VMM, SRS, Micro-pattern gaseous detectors, FPGA, Readout system

\section{Introduction}

Most radiation detectors, not only in the field of particle physics, are based on the principle of reading out charge signals. To obtain spatial information about the incident radiation, they include separated detection elements and

5 hence have many readout channels. High density electronics is required in order to process charge signals by amplification, shaping and digitisation. In the field of Micro-Pattern Gaseous Detectors [1] (MPGDs), strip, pixel or pad anodes are used to collect the amplified charge of ionising particles traversing a gas volume. Because of the small size of detector elements, integrated circuits are used and mounted close to the detector to process charge signals. Several Application Specific Integrated Circuits (ASICs) have been designed for different applications. The RD51 collaboration, which facilitates the development of these types of detectors, has developed a multi-purpose readout system, the Scalable Readout System [2] (SRS), in which many different ASICs can be implemented.

Within the BrightnESS project [3] connected to the European Spallation Source ERIC (ESS) [4, one such ASIC, the VMM [5] developed by Brookhaven National Laboratory (BNL), is implemented in the system. The VMM is designed for the ATLAS New Small Wheel project for the upgrade of parts of the muon system, where it is used to read out Micromegas [6] and small-strip Thin 20 Gap Chambers (sTGC) 7].

In our contribution to BrightnESS, the VMM serves as readout for a neutron GEM [8] detector based on a gadolinium converter [9] for the NMX instrument [10]. The SRS readout chain will be capable to cope with the high data rates [11. Besides of this application, the system is already used for develop- 
ments in the framework of the ALICE FoCal-E Pad detector [12.

\section{The Scalable Readout System}

Developed by the RD51 Collaboration since 2009, the SRS features high flexibility, uses commercially available components and presents a complete solution for detector readout, starting from minimal table top systems and scaling up to large rack-based systems. In contrast to application specific readout systems, SRS represents a general-purpose approach, consisting of a common back-end and detector specific front-end, which has to be adapted to the specific needs of the experiment it shall be used for. This approach reduces costs and manpower, as only the user-specific part of the system has to be developed for a new application.

A schematic drawing of the complete electronics chain is shown in Figure 1 It starts at the detector side with the so-called hybrid, a user-specific frontend ASIC on a printed circuit board (PCB) attached to the charge detecting structure of the detector. A number of different front-end ASICs have been 40 implemented in the SRS, for instance the APV25 [13, the Timepix [14] or the Beetle [15]. HDMI cables connect the hybrid to the core of the SRS: a userspecific adapter card connected to the SRS common Front-End Concentrator (FEC, see Section 2.3). A total of eight hybrids can be connected to one adapter card. A Gigabit Ethernet connection between the FEC and a computer is established either directly or via an Ethernet switch. The components of this local network using standard Ethernet UDP communication are several FECs, each with a unique IP and the computer. User-specific data acquisition and slow control software is used to operate the SRS. Optionally instead of a switch, a Scalable Readout Unit (SRU) is available, in which the data of up to 40 FECs are combined. The scalability of the system is achieved by the fact that several hybrids can be connected to one FEC and several FECs can be connected to a computer. 


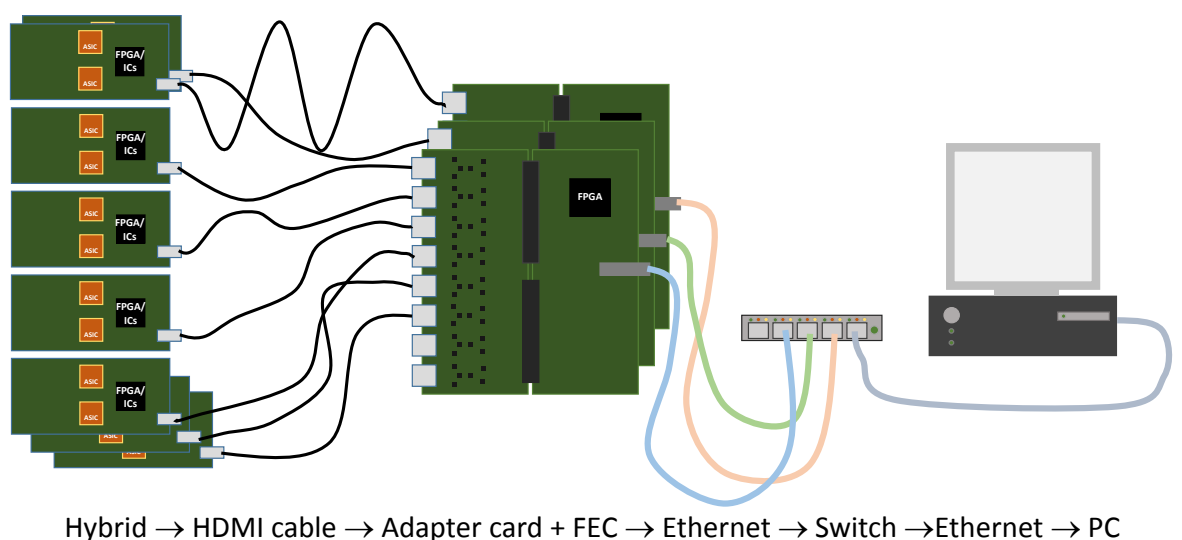

Figure 1: Schematic of the electronics for the SRS readout.

\subsection{The Hybrid}

The interface between detector and readout electronics is the hybrid. It connects a specific readout ASIC to the detection elements and provides the input needed by the chip as for example power, clock and slow control signals and transmits the data to the adapter card.

\subsection{The adapter card}

As the hybrid, the design of the adapter card is specific to the front-end ASIC employed. By default, the connection to the hybrids is provided by HDMI cables for most implementations. Depending on the type of front-end technology (analogue, digital, pixel ASIC), the components and logic on the adapter card may vary significantly. The adapter card connects to the FEC via one or two PCI connectors depending on its size and the specific implementation.

\subsection{The Front-End Concentrator card}

As core of the back-end, the FEC [16] provides communication with a computer by Gigabit Ethernet. The FEC card front-panel implements $2 \times$ RJ45 for trigger and external clock, $2 \times \mathrm{SFP}$ as data links, $2 \times$ LEMO-50 $\Omega$ trigger 
in- and outputs, $1 \times$ differential multi-purpose input and a 9-way connector for

power as can be seen in Figure 5 in Section 4.2. Up to eight FECs can be housed in a 19-inch ATX-powered Eurocard chassis.

The FEC card holds power converters, flash memory, a slot for a DDR3 memory and a Virtex-6 FPGA [17] . The firmware of the FEC is individual for different types of front-ends.

75 3. The VMM ASIC

For the readout of the NSW detectors, a new front-end ASIC, the VMM is developed by BNL. The design is driven by the detector characteristics and the timing and resolution requirements given by the physics and trigger scheme of the ATLAS experiment. The first version of the ASIC was available 2012 and extensively tested with different detectors [5, 18, 19]. After the second version VMM2 20] in 2014 and VMM3 in 2016, first quantities of the final ASIC VMM3a have become available at the beginning of 2018.

The VMM ASIC is designed in $130 \mathrm{~nm}$ technology and has 64 input channels, each with a preamplifier, shaper, a peak detector and several ADCs. The digitised data set of 38 bits per hit is multiplexed and can be read out on two lines double data rate with a clock frequency of up to $200 \mathrm{MHz}$. The chip can be programmed via two digital lines (data and clock).

As in the application in the NSW, positive as well as negative charge will be detected, the analogue part is designed for both polarities. Due to the different charge collection elements such as pads, anode strips and wires, detector capacitances between a few $\mathrm{pF}$ and $3 \mathrm{nF}$ can be handled. The input charge acceptance ranges from $0.1 \mathrm{pC}$ to $2 \mathrm{pC}$ with a resolution smaller than $1 \mathrm{fC}$ rms. A time resolution below one nanosecond is foreseen.

The chip is designed with low power consumption and can be programmed for a wide range of applications due to an adjustable gain, peaking time, polarity, threshold and timing precision. It includes a dedicated test pulse circuit per channel and single-channel threshold trimming. 
Only the arrival time (time at the peak or time of threshold crossing) and amplitude of the analogue input signal are measured, digitised and multiplexed with the number of the channel hit into a data stream. For triggering, the channel number of the first hit is provided with a minimal delay (address in real time, ART). Additionally for each channel, a direct and fast digital output signal can be generated, which can be the Time over Threshold (ToT), Time to Peak (TtP), Peak to Threshold (PtT), Peak to Peak (PtP) or a 6-bit low-resolution $\mathrm{ADC}$ value of the amplitude with a conversion time of $25 \mathrm{~ns}$.

Figure 2 illustrates the treatment of an analogue signal over threshold in the VMM in case of a configuration to measure the arrival time at the peak position. To measure the amplitude, the Peak Detector Output (PDO) signal rises with the input pulse. When the peak is detected, PDO stays at this level and the amplitude is later digitised by a 10-bit ADC.

For timing, a voltage ramp of the Time Detector Output (TDO) starts to increase linearly from the time of the peak until the next falling edge of a clock. The same clock also increments a 12-bit counter, which serves as coarse timing. For the fine timing between clock cycles, the value of TDO is digitised by an 8-bit ADC.

The VMM has an option to enable the readout of data from channels, for which the threshold has not been reached, but which are adjacent to a channel with a signal over threshold, a so called neighbouring logic. This way, shared charge can still be detected, even though it is not enough to surpass the thresh120

After the conversion time (in the order of $250 \mathrm{~ns}$ ), the channel is reset and the data set (38 bits per hit) is latched onto a four hit deep buffer in continuous mode. Theoretically, the per channel hit rate reaches up to $4 \mathrm{MHz}$, which however can be reduced by the return to baseline in the analogue part of the 125 ASIC. By using a token scheme, the data set is pushed out of the VMM on two data lines by another clock (CKDT). 


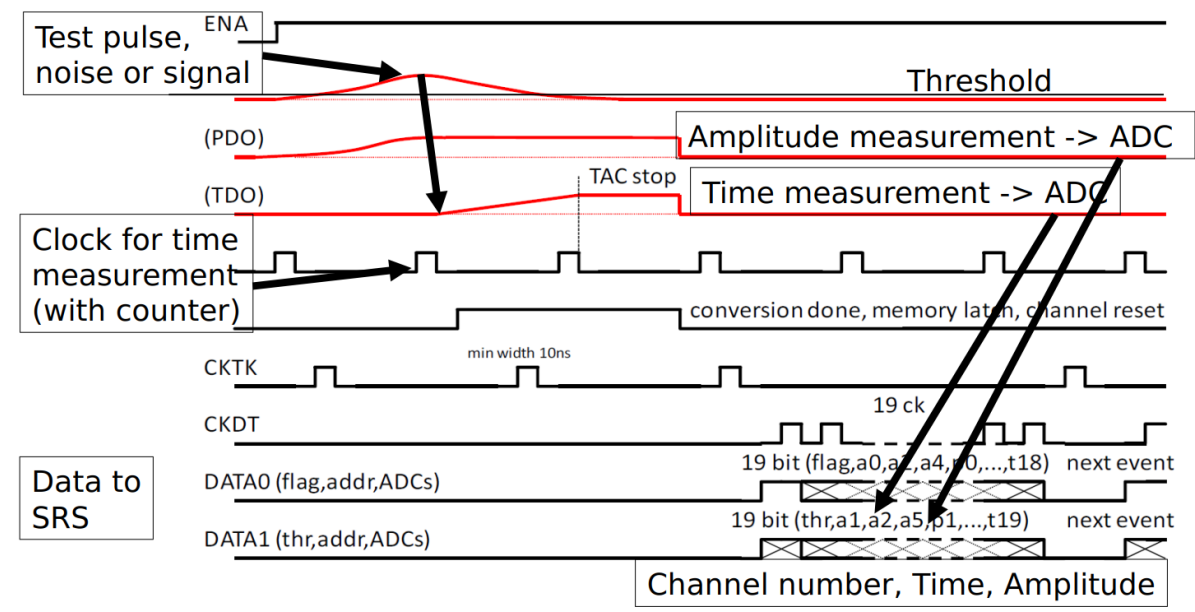

Figure 2: Processing of an analogue pulse in the VMM continuous mode, modified from 21.

\section{SRS + VMM developments}

In a similar way as for the other ASICs, the VMM chip has been implemented in the SRS. Following the SRS requirements to implement a new chip, FPGA firmware, an adapter card and a hybrid to be connected to the detector have been designed. These components are explained in this section.

\subsection{RD51 VMM hybrid}

To implement the VMM in the SRS, the front-end ASIC is placed on a hybrid, which is the first part of the electronics chain and directly connected to the detector. Each input channel is spark-protected via a TVS diode IC [22] and AC-coupled to an input of the VMM, see Figure 3

For interchangeability with the established APV25 hybrid, two VMM ASICs are placed on one VMM hybrid as can be seen in Figure 4, such that 128 channels can be read out as with the APV25. The PCB also holds an FPGA of type Spartan-6 23] to combine the signals of both VMM ASICs and distribute the slow control data. This FPGA also generates the necessary clocks and control signals to operate the VMMs. Besides ASICs and FPGA, the top side of the hybrid includes a flash memory for FPGA configuration, mini HDMI and 


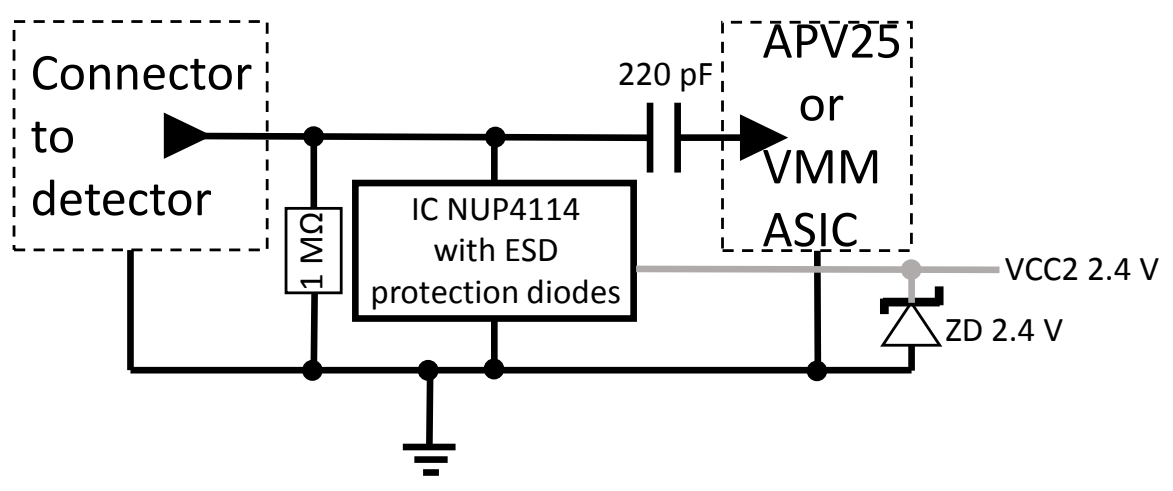

Figure 3: Protection circuit of the APV25 and VMM hybrid for the ASIC input channels.

external power connectors and the connector to the detector. LDOs for power supply are placed at the bottom side. In the current version of the hybrid, the

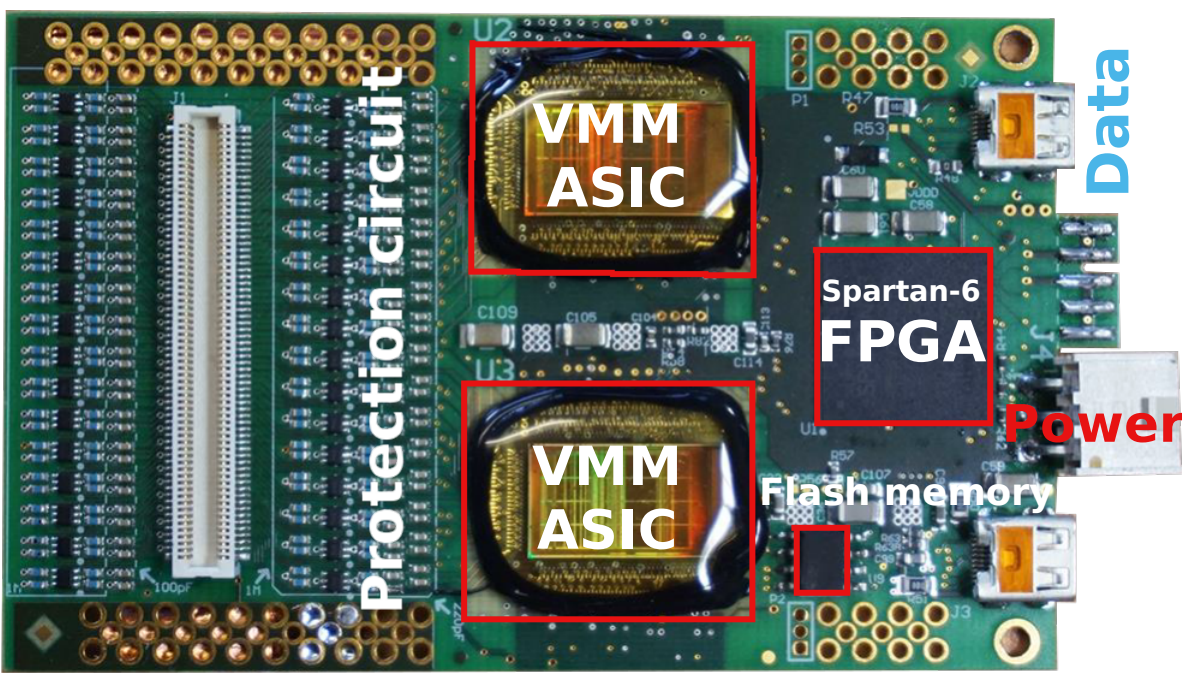

Figure 4: VMM3 hybrid of the Scalable Readout System

direct outputs of the VMMs are not connected and only the multiplexed data output are used. The routing of the input signal lines from the connector is implemented in such a way that neighbouring detector channels are connected to neighbouring input channels of the VMMs. To allow for an application of the neighbouring logic over the complete detector, inter-ASIC signals are con- 
nected between the two VMMs and are accessible on the PCB for inter-hybrid connection.

The FPGA on the hybrid is connected to the VMMs to provide input clocks, configuration and slow control signal and receive the multiplexed output data. It is also connected to the mini HDMI connector for communication with the adapter card and hence the FPGA on the FEC.

These signals are a master clock, slow control data and I2C. In addition, lines of the HDMI cable can used to power the hybrid from the adapter card. Another option is to connect an external power supply directly to the hybrids.

Two supply voltages for the LDOs are necessary, one for a circuit with the FPGA and flash memory and a second one for the VMM ASICs. They require at least $2.6 \mathrm{~V}$ and $1.3 \mathrm{~V}$, respectively. The LDOs can withstand at most $3.6 \mathrm{~V}$. The measured average supply currents are $0.45 \mathrm{~A}$ (at $3.0 \mathrm{~V}$ ) and $1.3 \mathrm{~A}$ (at $1.8 \mathrm{~V}$ ), respectively without a significant increase during data acquisition.

Due to the power consumption of the VMMs and the resulting voltage drop, it is not possible to use the HDMI powering option with cables longer than $3 \mathrm{~m}$ in order to stay within the margins of the LDO supply voltages in both cases of switching on and operation.

\subsection{Adapter card}

Figure 5 shows the combination of an SRS FECv_6 and the adapter card for the VMM hybrids. The card has eight HDMI type A plugs to connect to the hybrids. The adapter card mainly holds LVDS drivers, as the data from the VMM hybrid are already digital and no further processing is required. All components are powered by the FEC. For direct powering of the VMM hybrids a SATA connector is placed on the side of the adapter card facing the FEC. A converter produces the voltages required by the hybrids, which are tunable by potentiometers to allow for a compensation of the voltage drop in the cables.

\subsection{Firmware}

To implement the VMM in the SRS, a new firmware for the Virtex-6 FPGA

on the FEC was required. Many common parts of the general SRS firmware 


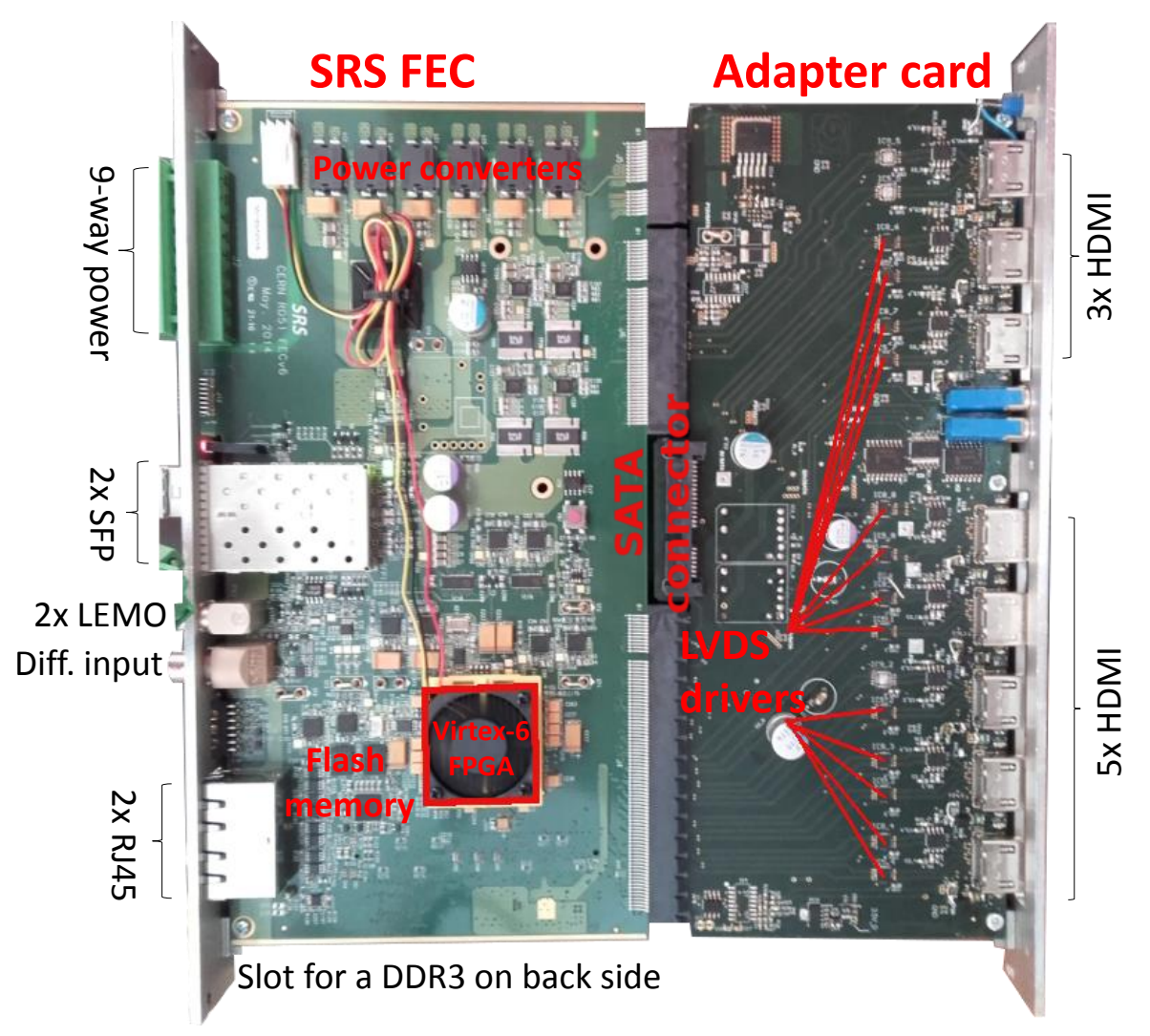

Figure 5: FEC (left) and adapter card (right) for the SRS VMM system. 
could be reused, such as the Ethernet MAC and package analysers, reset logic, clocking resources and configuration logic. The front-end specific part is designed to treat large amounts of data. It consists of two basic components. The first one is designed for the control and configuration of the ICs on the adapter card and hybrid, while the second one processes the data from the hybrid. The second part also includes an decoder, as the data between hybrid and FEC are transferred 8b10b decoded.

\subsection{Electronics chain}

The SRS standard readout chain shown in Figure 1 has been realised in a small-scale prototype, but already complete chain. All hardware components of the schema are realised and presented in Figure 6 (two hybrids connected in this case) with the addition of the SRS crate for power supply of the FEC card and adapter card. For such a small system, the hybrids are powered by the adapter card through the HDMI cables.

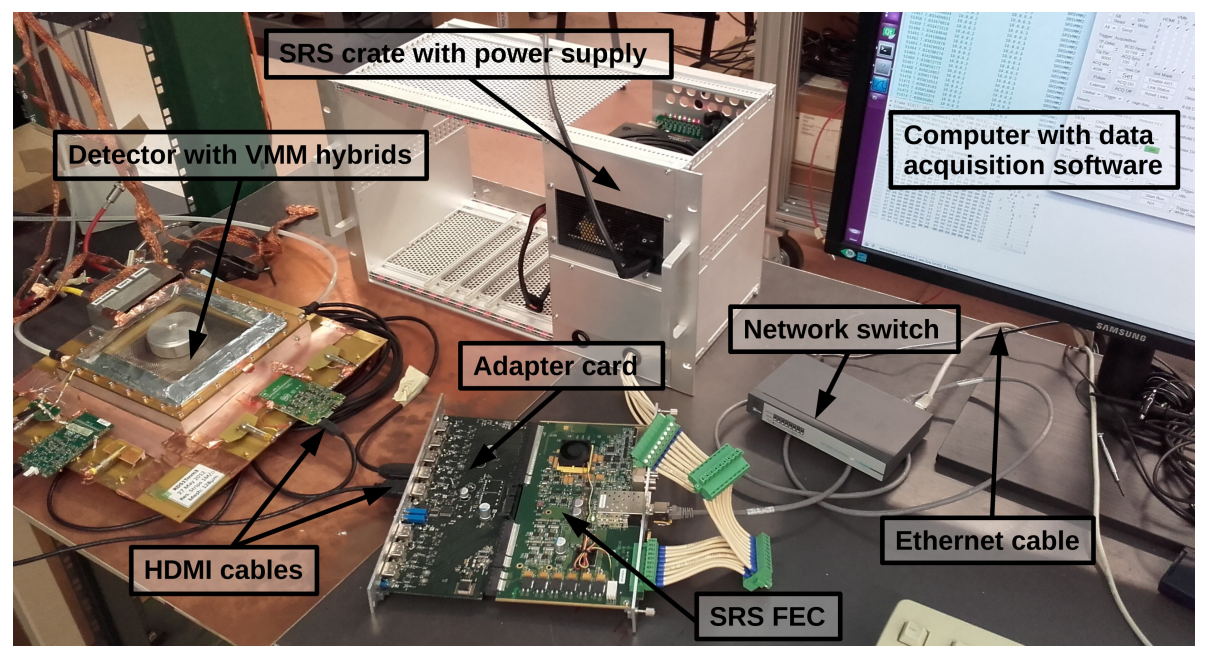

Figure 6: Prototype electronics chain in a laboratory setup. 


\subsection{Software}

Several software packages are under development in order to control the readout system and the VMMs, read out and store the data and permit online monitoring. As a starting point for the slow control, software provided by the ATLAS NSW upgrade project was used to develop an adapted software based on front-end ASIC, as such large amounts of data will usually not occur in all VMMs simultaneously and continuously.

To cope with large amounts of data simultaneously occurring in all VMMs of a system, buffers in the VMMs, Spartan- 6 on the hybrid and Virtex-6 on the advantage of the scaled system pays off again, as such excesses are compensated by other VMMs with lower data rate and only an average amount of data needs to be processed. 
64 channels, a VMM ASIC can produce 9.7 Gbit/s of data. By design, this large amounts of data cannot be read out from the ASIC, as the maximum readout clock for the two data output lines is $200 \mathrm{MHz}$. In double data rate mode, the readout rate can reach $800 \mathrm{Mbit} / \mathrm{s}$ equivalent to $21 \mathrm{Mhits} / \mathrm{s}$ per VMM.

230 from both VMMs and hence can receive up to $1.6 \mathrm{Gbit} / \mathrm{s}$. For the transmission through the HDMI cables, one differential pair per VMM is used with a serialiser logic in the FPGA that can drive up to $950 \mathrm{Mbit} / \mathrm{s}$. As the data stream between hybrid and FEC 8b10b encoded, this is almost sufficient to transfer the complete maximum data stream from both VMMs.

HDMI cables come in two different categories. Cat 2 cables are suitable as they are specified for $340 \mathrm{MHz}$ and work well for frequencies up to $825 \mathrm{MHz}$.

The deserialisers of the Virtex-6 on the FEC are designed for up to $1.1 \mathrm{GHz}$, which means the FPGA can receive data at maximum speed. It can serialise at frequencies up to $2.7 \mathrm{GHz}$ to transmit data, which theoretically is the maximum amount of data of 3.375 VMMs. The Gigabit Ethernet between FEC and computer, however, limits the theoretical maximum data rate to 1 Gbit/s. Solution to overcome this bottle neck are presented in Section 6

\section{Basic system tests}

Basic tests of the system were performed using the analogue monitoring outputs of the VMMs on the hybrid. First, the correctness of configuration of the VMMs was verified. The analogue monitoring output was programmed to display the threshold voltage level. The results of this measurement for different DAC settings are displayed in Figure 7. The relation between the setting of the measurement for one specific VMM hybrid proves that the configuration data stream is uploaded correctly. In case of a bit shift, steps would be visible in the measured curve. 


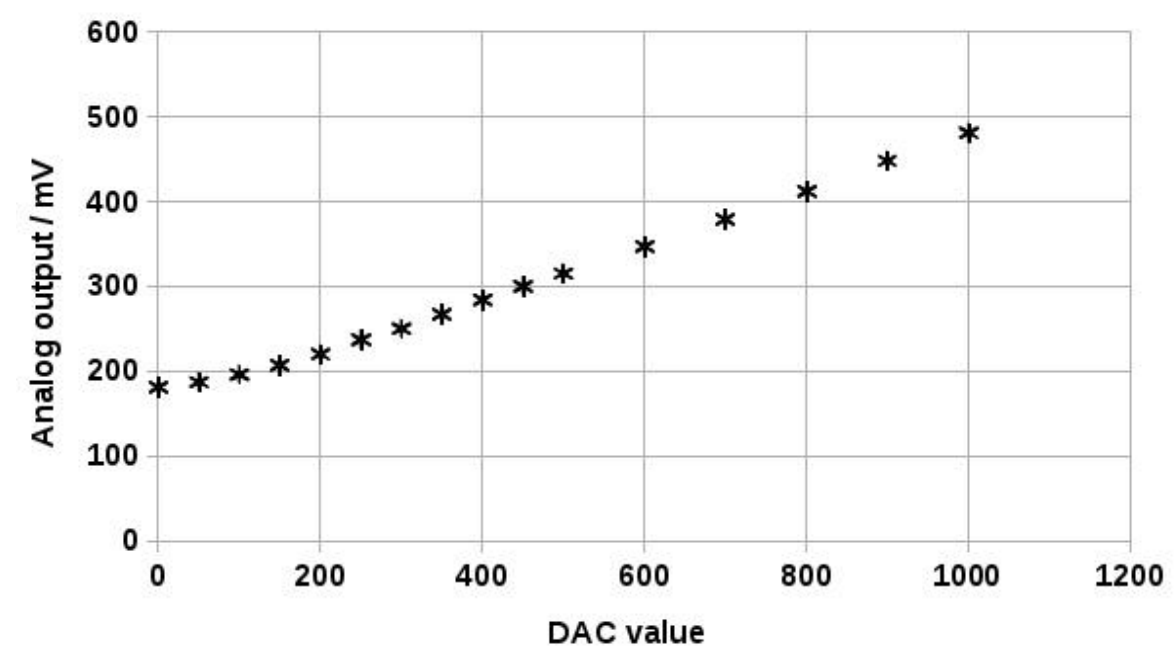

Figure 7: Analogue output voltage in dependence of the configured threshold.

In further tests, the threshold level of individual channels was monitored and the effect of the single-channel trim was quantified. The result of this measurement was as expected a linear decrease of the threshold level with the trim, except for the highest values due to a known design error of the VMM. Also, the pedestal of every individual channel was investigated showing variations between different channels, what has also been observed already by colleagues from ATLAS NSW 21. An internal test pulse was activated on the channel connected to the monitoring output. Different pulser DAC values, peaking times, gains and polarities were investigated. As an example, Figure 8 shows the digitised waveform of two test pulses (generated in each of the two VMMs on the hybrid) with the same configuration except for the peaking time (25 ns and $200 \mathrm{~ns})$. This proofs that both VMMs on a hybrid can be configured individually.

To test the detector input channels, externally generated test pulses were used. A charge signal was generated by a voltage drop on a capacitor with an arbitrary function generator. Again by using the monitoring output, the shaped and amplified signal could be observed proving that the configuration of the analogue part of the ASIC and the board design was done correctly. 


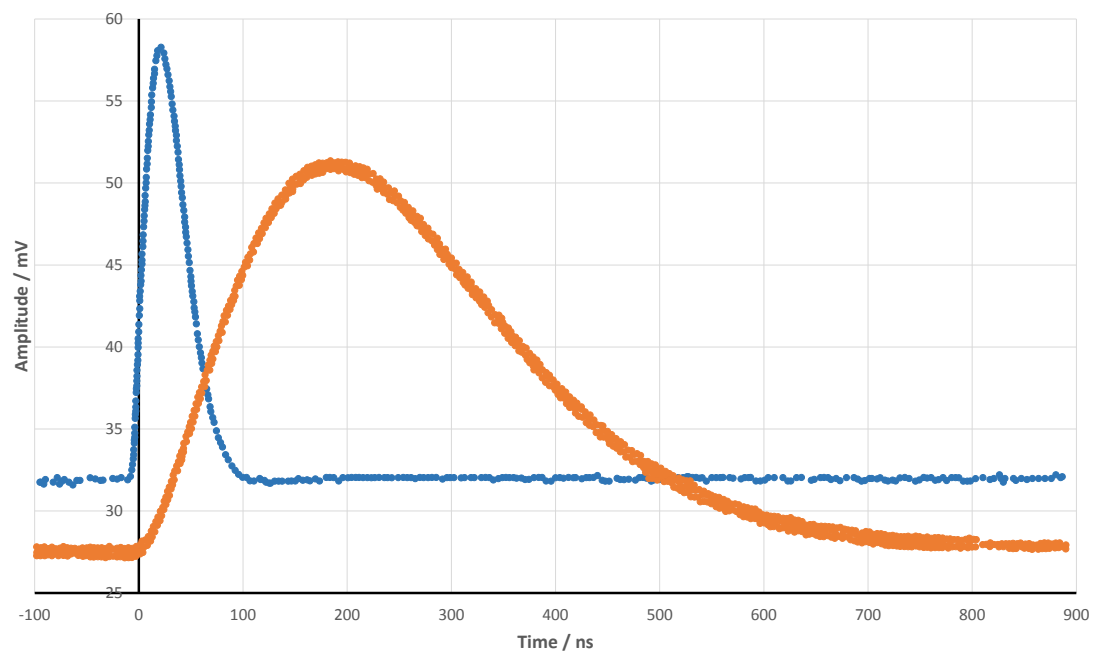

Figure 8: Test pulses with peaking times of $25 \mathrm{~ns}$ and $200 \mathrm{~ns}$.

Next, data received in the VMM induced by test pulses were investigated by using the Xilinx Chipscope software 25$]$ to test the readout chain. Figure 9 shows the monitored signals in the Spartan-6 FPGA on a hybrid, when data from two VMMs are received. For one VMM a single channel was pulsed, while for the second one, two channels have been activated. For each VMM, the data set is received on the two output lines with 19 bit on each, proving that the digitisation works correctly.

\begin{tabular}{|c|c|c|}
\hline & $1^{\text {st }}$ test pulse & $2^{\text {nd }}$ test pulse \\
\hline VMM0_data_0 & ロレロロ 19 bit & \\
\hline VMM0_data_1 & $几 19$ bit & \\
\hline VMMO_Data_clock & 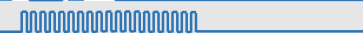 & \\
\hline VMMO_writēToFIFO & $1^{\text {st }}$ written hit to FIFO & \\
\hline VMM1_data_0 & ロபレபレロ 19 bit & உロூ几 \\
\hline VMM1_data_1 & பேபாட 19 bit & ロ几几 \\
\hline VMM1_Data_clock & 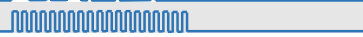 & 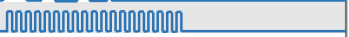 \\
\hline VMM1_writeToFIFO & $1^{\text {st }}$ written hit to FIFO & $2^{\text {nd }}$ written hit to FIFO \\
\hline
\end{tabular}

Figure 9: Data received in the Spartan-6 FPGA on a VMM hybrid monitored with the Xilinx Chipscope software. Test pulses have been applied to the two VMMs on the hybrid. VMM0_data_0 and VMM0_data_1 are the data of one VMM with a single test pulse. VMM1_data_0 and VMM1_data_1 are the data of the second VMM with two test pulses. 
To further test the readout chain, the path of the hit information through the system was checked using Chipscope on the Spartan-6 on the hybrid as well as on the Virtex-6 FPGA on the FEC.

Finally, the data stream arrives at the network interface of the computer, which can be monitored with the Wireshark software [26], which proves that the complete readout chain works correctly. Figure 10 shows a screen shot of the software display. The individual Ethernet packages exchanged between FEC and computer are displayed at the top part. In the lower section of the window, the content of one package from the FEC with data of one VMM is shown. A plugin is used to display the data in a readable way. The insert shows the individual hits with the information about the channel coarse time (BCID), precise time (tdc) and amplitude (adc).

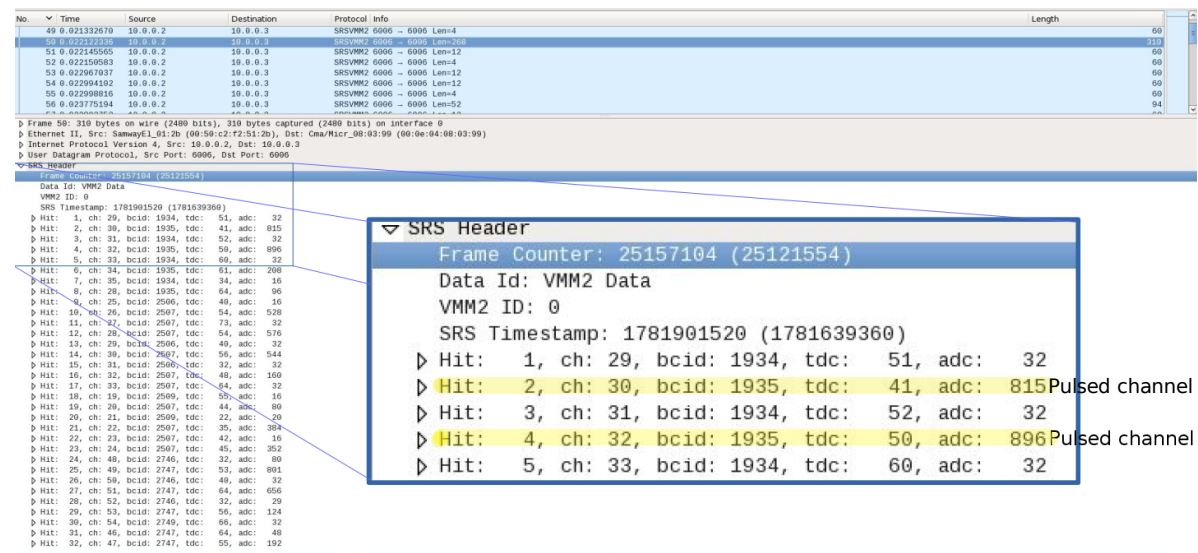

Figure 10: Monitoring of the data received at the network card of the computer connected to a FEC with VMMs using the Wireshark software. A single Ethernet package is selected and the data shown in the bottom part of the figure. A plugin is used to convert the binary information to the measurement results of the VMM.

This data has been taken in a configuration where every second channel was pulsed as can be seen from the high adc value for the even channels. Despite their adc value is lower than the threshold was set the odd channels are also read out, since the neighbouring logic was enabled in this configuration and the data verifies that it works correctly. 
After verifying the functioning of the readout chain, triple-GEM-based detectors were tested in pion or muon beams provided in beam lines of the SPS at CERN after having tested the setup in the laboratory using a ${ }^{55} \mathrm{Fe}$ source. A first analysis result of the data rate in a pion beam is shown in Figure 11 . The rate of particles in this setup with two VMM hybrids ( $\mathrm{x}$ and $\mathrm{y}$ strip readout) is on the same order as needed for the application at the NMX instrument.

The noise level of the VMM hybrid is in the order of 800 electrons when no external detector capacitance in connected. A more detailed study of the noise in dependence on configuration parameters and detector capacitance is ongoing.

The VMM readout has also been used together with a small-scale prototype for the BrightnESS project based on a gadolinium converter with triple-GEM amplification stage at a neutron source. Results of those measurements will be presented in future publications.

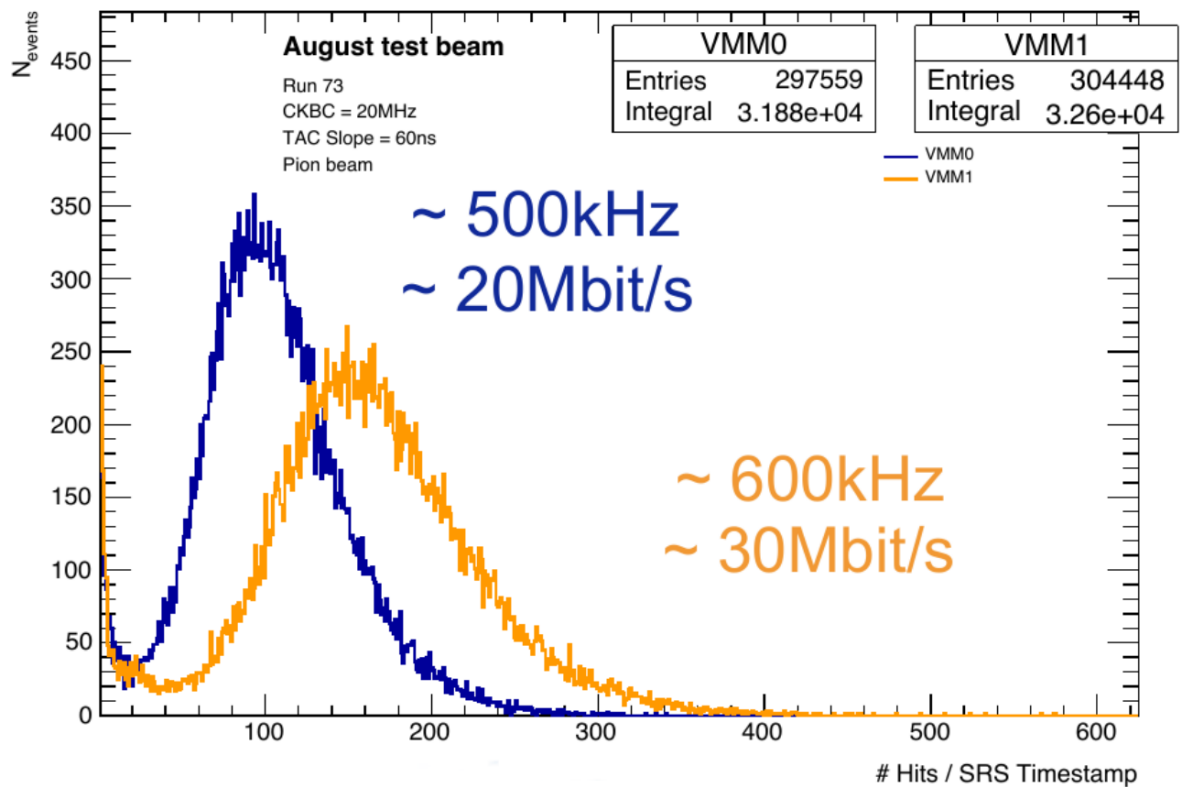

Figure 11: Number of readout hits on strips per readout cycles of $5 \mathrm{kHz}$ for two VMM hybrids (VMM0 and VMM1) in a test beam setup with a triple-GEM detector in a pion beam. One SRS Timestamp is equivalent to one readout cycle, which was triggered periodically with 5 $\mathrm{kHz}$. 


\section{Upgrades}

The implementation of the VMM ASIC in the SRS is still ongoing. A pro-

310 continuously improved. The firmwares for the Spartan-6 and Virtex-6 FPGAs still allow for improvements to achieve the maximum data rates the hardware components of the system are designed for.

A significant upgrade, which will enable reading out more VMMs hybrids to the one implemented for the APV25. Instead of a single VMM hybrid, two hybrids will be connected to a single HDMI input on the adapter card. However, due to the large amounts of data, the implementation will be different compared to the one in the case of the APV25, where data streams from the slave hybrid be identical and send their data streams through their own HDMI cable to a splitter, which combines the data into a single HDMI cable, connected to the adapter card. More details on this scheme can be found in [27.

As can be seen from the calculations in Section 4.6, the Gigabit Ethernet would be to use 10 or 100 Gigabit Ethernet instead in order to stick to standard network components. However, the Virtex-6 FPGA on the FEC does not allow such an upgrade, as it is not specified for higher bandwidths. To overcome this issue, data reduction could be implemented in the FPGA. In case of the method [28].

Another solution would be to revise the FEC. Due to the large effort of a redesign of the FEC, the short timescale solution of a workaround as explained below is currently followed. Instead of Gigabit Ethernet, Serial Rapid IO (SRIO) 335 is studied, which can be implemented with the maximum $2.7 \mathrm{GHz}$ output clock and several lines. The data from the FEC will be transferred to another board with a System on Chip (SoC) for data reduction and 10 or 100 Gigabit Ethernet 
output.

\section{Conclusion}

340 the Scalable Readout System of the RD51 collaboration. Dedicated hardware components have been designed and produced and FPGA firmware as well as operating software has been developed. The complete detector readout chain is operational and data from micro-pattern gaseous detectors with strip readout have been obtained both in laboratory as well as beam tests. The functioning of the individual parts of the readout chain has been verified using VMM internal signals available at the monitoring output like the pedestals or threshold levels as well as internal test pulses. Correctly digitised data arriving at the computer have been monitored. Even in a prototype state, the system can be operated

\section{Acknowledgements}

We would like to thank our colleagues of the ATLAS NSW electronics group, especially George Iakovidis, for the fruitful discussions and exchange of information regarding the VMM. Gratitude also goes to our collaborators in WP5 355 of the BrightnESS project and at the European Spallation Source ERIC. This work was supported in part by the European Unions Horizon 2020 research and innovation programme under grant agreement No. 676548 (the BrightnESS project).

\section{References}

[1] S. Levorato, S. Dalla Torre, MPGD: new developments and applications PoS (2016) 012.

[2] S. Martoiu, H. Muller, J. Toledo, Front-end electronics for the Scalable Readout System of RD51, in: Nuclear Science Symposium and Medical 
Imaging Conference (NSS/MIC), 2011, p. 2036. doi:10.1109/NSSMIC.

[3] BrightnESS webpage.

[4] European Spallation Source ESS ERIC webpage.

[5] G. D. Geronimo, J. Fried, S. Li, J. Metcalfe, N. Nambiar, E. Vernon, V. Polychronakos, VMM1 - An ASIC for micropattern detectors, in: 2012 IEEE Nuclear Science Symposium and Medical Imaging Conference Record (NSS/MIC), 2012, pp. 633-639. doi:10.1109/NSSMIC.2012.6551184.

¿ [6] Y. Giomataris, P. Rebourgeard, J. Robert, G. Charpak, Micromegas: a

口. high-granularity position-sensitive gaseous detector for high particle-flux environments, Nuclear Instruments and Methods in Physics Research Section A 376 (1) (1996) 29 - 35. doi:10.1016/0168-9002(96)00175-1.

[7] E. Perez Codina, Small-strip Thin Gap Chambers for the muon spectrometer upgrade of the ATLAS experiment, Nucl. Instrum. Meth. A824 (2016) 559-561. doi:10.1016/j.nima.2015.11.095

[8] F. Sauli, Gem: A new concept for electron amplification in gas detectors Nuclear Instruments and Methods in Physics Research Section A 386 (2-3) (1997) 531 - 534. doi:10.1016/S0168-9002(96)01172-2.

[9] D. Pfeiffer, P. Thuiner, D4.3 - Natural and enriched Gadolinium converters in: BrightnESS Deliverable Report, 2016, pp. 5-18.

[10] S. Peggs, et al., ESS Technical Design Report, in: ESS-2013-0001, 2013.

[11] M. Lupberger, D4.9 detector electronics chain, in: BrightnESS Deliverable Report, 2017.

[12] B. Kim, T. Chujo, M. Inaba, H. Muller, G. Iakovidis, E. Oliveri, 19pAA-

a 11 Examining the SRS VMM2 based hybrid as a front-end board for the ALICE FoCal-E Pad detector, Meeting Abstracts of the Physical Society of Japan 71 (2016) 292. doi:10.11316/jpsgaiyo.71.1.0_292. 
[13] S. Martoiu, H. Muller, A. Tarazona, J. Toledo, Development of the scalable readout system for micro-pattern gas detectors and other applications, Journal of Instrumentation 8 (03) (2013) C03015.

[14] X. Llopart, et al., Timepix, a 65k programmable pixel readout chip for arrival time, energy and/or photon counting measurements., Nuclear Instruments and Methods in Physics Research Section A 581.1 (2007) 485 494, Erratum ibid. A 585 (2008) 106. doi:10.1016/j.nima.2007.08.079

[15] M. Agari, et al., Beetle - a radiation hard readout chip for the LHCb experiment, Nuclear Instruments and Methods in Physics Research Section A 518 (1) (2004) 468 - 469, Frontier Detectors for Frontier Physics: Proceeding. doi:10.1016/j.nima.2003.11.058.

[16] J. Toledo, H. Muller, R. Esteve, J. Monzó, A. Tarazona, S. Martoiu, The front-end concentrator card for the RD51 scalable readout system, Journal of Instrumentation 6 (11) (2011) C11028. doi:10.1088/1748-0221/6/11/ C11028.

[17] Xilinx, Inc., Virtex-6 FPGA Data Sheet: DC and Switching Characteristics, DS152 (v3.6), 20,25 (March 2014).

[18] The ATLAS collaboration, Performance of the First Version of VMM Front-End ASIC with Resistive Micromegas Detectors, Tech. Rep. ATLUPGRADE-PUB-2014-001, CERN, Geneva (Sepember 2014).

[19] G. Iakovidis, V. Polychronakos, G. De Geronimo, VMM - An ASIC for Micropattern Detectors, Tech. Rep. ATL-MUON-PROC-2015-015, CERN, Geneva (Nov 2015).

[20] G. D. Geronimo, VMM2 - An ASIC for the New Small Wheel, in: Presentation given at TWEPP 2014, 2014.

[21] G. Iakovidis, personal communication, 2016. 
[22] ON Semiconductor, NUP4114 Series, Low Clamping Voltage ESD Protection Diode,

[23] Xilinx, Inc., Spartan-6 FPGA Data Sheet: DC and Switching Characteristics. DS162 (v3.1.1), 18, 56. (January 2015).

[24] Apache Software Foundation, Apache Kafka: A distributed streaming platform (January 2016).

[25] Xilinx, Inc., Xilinx ChipScope Pro Software and Cores User Guide (UG029)

[26] Wireshark Foundation, Wireshark User's Guide.

[27] M. Lupberger, VMM and the SRS - update, in: Presentation given at the RD51 Collaboration Meeting at CERN, 2017, pp. 18-21.

[28] D. Pfeiffer, et al., The $\mu$ TPC method: improving the position resolution of neutron detectors based on MPGDs, Journal of Instrumentation 10 (2015) P04004. 